\title{
PROOF OF AN ARITHMETIC THEOREM DUE TO LIOUVILLE.
}

BY PROFESSOR E. T. BELL.

1. The theorem, quoted from volume II, page 337, of Dickson's History of the Theory of Numbers, is as follows. If $f(m), F(m)$ are two arbitrary functions having definite values for $m=1,2,3, \cdots$, and

$$
X_{\mu}(m)=\Sigma d^{\mu} f(d), \quad Z_{\mu}(m)=\Sigma d^{\mu} F(d),
$$

where each summation extends over all divisors $d$ of $m$, then for any real or complex numbers $\mu, \nu$, and $\delta=m / d$, we have

$$
\Sigma d^{\mu-\nu} X_{\nu}(d) Z_{\mu}(\delta)=\Sigma d^{\mu-\nu} Z_{\nu}(d) X_{\mu}(\delta) .
$$

No reference to a proof being given, presumably none has been published. But Liouville remarks* that "there is an exceedingly simple method which I shall develop on another occasion, and which will lead us very rapidly by a kind of regular and general algorithm to the formula $(A)$." Without going into more detail than suffices for the proof of $(A)$ we shall indicate the nature of an algorithm of this type. Arbitrary functions such as $f, F$ which take definite values for integral arguments are called numerical functions All of the functions considered in this paper are of this kind.

2. Let $(d, \delta)$ denote any pair of conjugate divisors of $m$. Form the value of $\varphi_{1}(x) \psi(y)$, where $\varphi_{1}$ and $\psi$ are arbitrary functions, for $(x, y)=(d, \delta)$, sum $\varphi_{1}(x) \psi(y)$ over all pairs $(d, \delta)$, and denote the result by $\Sigma_{m} \varphi_{1}(d) \psi(\delta)$. Let $\left(\delta_{1}, \delta_{2}\right)$ denote any pair of conjugate divisors of $\delta$, so that $m=d \delta, \delta=\delta_{1} \delta_{2}$, $m=d \delta_{1} \delta_{2}$, and put $\psi(m)=\Sigma_{m} \varphi_{2}(d) \varphi_{3}(\delta)$; whence

$\Sigma_{m} \varphi_{1}(d) \psi(\delta)=\Sigma_{m}\left[\varphi_{1}(d) \Sigma_{\delta} \varphi_{2}\left(\delta_{1}\right) \varphi_{3}\left(\delta_{2}\right)\right]=\Sigma_{m} \varphi_{1}\left(d_{1}\right) \varphi_{2}\left(d_{2}\right) \varphi_{3}\left(d_{3}\right)$ the last summation extending over all triads $\left(d_{1}, d_{2}, d_{3}\right)$ of divisors defined by $m=d_{1} d_{2} d_{3}$. If now $(p, q, r),(i, j, k)$ are any permutations of $(1,2,3)$, it is obvious that

$$
\Sigma_{m}\left[\varphi_{p}(d) \Sigma_{\delta} \varphi_{q}\left(\delta_{1}\right) \varphi_{r}\left(\delta_{2}\right)\right]=\Sigma_{m} \varphi_{i}\left(d_{1}\right) \varphi_{j}\left(d_{2}\right) \varphi_{k}\left(d_{3}\right)
$$

which may be written in the purely symbolic form

$$
\varphi_{p} \cdot \varphi_{q} \varphi_{r}=\varphi_{i} \varphi_{j} \varphi_{k}
$$

* Liouville, Journal des mathématiques, (2), vol. 3 (1858), p. 66. 
This can be extended to any number of symbolic factors. Thus for four numerical functions $\varphi_{1}, \varphi_{2}, \varphi_{3}, \varphi_{4}$, and $(p, q, r, s)$, $(i, j, k, l)$ any permutations of $(1,2,3,4)$, we have

$$
\varphi_{p} \varphi_{q} \cdot \varphi_{r} \varphi_{s}=\varphi_{i} \cdot \varphi_{j} \varphi_{k} \varphi_{l}=\varphi_{i} \cdot \varphi_{j} \varphi_{k} \cdot \varphi_{l}=\varphi_{i} \varphi_{j} \varphi_{k} \varphi_{l} \text {, etc. }
$$

In non-symbolic form, $\varphi_{p} \varphi_{q} \cdot \varphi_{r} \varphi_{s}$ is

$$
\Sigma_{m}\left[\Sigma_{d} \varphi_{1}\left(d_{1}\right) \varphi_{2}\left(d_{2}\right) \Sigma_{\delta} \varphi_{3}\left(\delta_{1}\right) \varphi_{4}\left(\delta_{2}\right)\right],
$$

the sums $\Sigma_{m}, \Sigma_{d}, \Sigma_{\delta}$ extending to all $(d, \delta),\left(d_{1}, d_{2}\right),\left(\delta_{1}, \delta_{2}\right)$, respectively, such that $m=d \delta, d=d_{1} d_{2}, \delta=\delta_{1} \delta_{2}$; and $\varphi_{i} \varphi_{j} \varphi_{k} \varphi_{l}$ is

$$
\Sigma_{m} \varphi_{i}\left(d_{1}\right) \varphi_{j}\left(d_{2}\right) \varphi_{k}\left(d_{3}\right) \varphi_{l}\left(d_{4}\right),
$$

the sum $\Sigma_{m}$ extending to all tetrads $\left(d_{1}, d_{2}, d_{3}, d_{4}\right)$ of divisors such that $m=d_{1} d_{2} d_{3} d_{4}$. The identity of these sums is obvious.

In precisely the same way the $\varphi^{\prime}$ 's in a product $\varphi_{1} \varphi_{2} \cdots \varphi_{n}$ of any number $n$ of symbolic factors are subject to the associative and commutative laws of formal algebra, and the interpretation of these laws for the general case in terms of multiple summations with respect to all the divisors of $m$ distributed into sets of $n$ or fewer each, is evident from the above illustrations.

3. Although it is not required for the proof of $(A)$, we may point out that if each $\varphi$ is such that $\varphi(1) \neq 0$, there is a unique division in this algebra. For it has been shown elsewhere* that for each $\varphi$ there is a unique $\varphi^{\prime}$ satisfying the equations

$$
\Sigma_{1} \varphi(d) \varphi^{\prime}(\delta)=1, \quad \Sigma_{m} \varphi(d) \varphi^{\prime}(\delta)=0 \quad(m>1) .
$$

Expressing this symbolically we write $\varphi \varphi^{\prime}=1$, or $\varphi^{\prime}=1 / \varphi$, and see that $\varphi_{i} \varphi_{j}=\varphi_{k}$ implies $\dot{\varphi}_{j}=\varphi_{k} \varphi_{i}{ }^{\prime}$, or in another form, $\varphi_{j}=\varphi_{k} / \varphi_{i}$. When the arithmetical or algebraic definition of $\varphi$ is given there is no difficulty in writing down the zeta function $\dagger$ which generates it. The zeta generator of $\varphi^{\prime}$ is obviously the reciprocal of the generator of $\varphi$.

* Bell, Tôhoku Mathematical Journal, vol. 17 (1920), p. 221.

$\dagger$ The zeta function generator of $\varphi$ is by definition the Dirichlet series in which the coefficient of $n^{-s}$ is $\varphi(n)$; viz. it is $\sum_{n=1}^{\infty} \varphi(n) / n^{s}$. For practically all the $\varphi(n)$ existing at present in arithmetic, $s$ can be chosen so that the series converge absolutely, and for an important class of $\varphi$ 's, those in which $\varphi(m n)=\varphi(m) \varphi(n)$ for $m, n$ relatively prime integers $>0$, the generator is decomposable into factors, etc., ef. Bachmann, Zahlentheorie, B. 2 Kap. 11. From these decompositions the relations between the $\varphi$ 's are developed isomorphically to the multiplicative properties of integers by means of the algorithm of this note. Such a course was evidently followed by Liouville in obtaining his results on numerical functions. 
4. Write $u_{k}(m)=m^{k}$, and put

$$
X_{k}{ }^{\prime}(m)=X_{k}(m) / m^{k}, \quad Z_{k}{ }^{\prime}(m)=Z_{k}(m) / m^{k} .
$$

Then from the definitions of the functions,

$$
X_{\mu}{ }^{\prime}=u_{-\mu} f, \quad Z_{\mu}{ }^{\prime}=u_{-\mu} F,
$$

and from the associative and commutative laws,

$$
u_{-\mu} F \cdot u_{-\nu} f=u_{-\mu} f \cdot u_{-\nu} F,
$$

we find $Z_{\mu}{ }^{\prime} X_{\nu}{ }^{\prime}=X_{\mu}{ }^{\prime} Z_{\nu}{ }^{\prime}$, which may be written in full as follows:

$$
\Sigma_{m} \frac{Z_{\mu}(\delta)}{\delta^{\mu}} \frac{X_{\nu}(d)}{d^{\nu}}=\Sigma_{m} \frac{X_{\mu}(\delta)}{\delta^{\mu}} \frac{Z_{\nu}(d)}{d^{\nu}} .
$$

Multiplying this throughout by $m^{\mu}$, we get $(A)$.

The University of Washington, November 30, 1920.

\section{A SEQUENCE OF POLYNOMIALS CONNECTED WITH THE $n$ TH ROOTS OF UNITY.}

BY DR. T. H. GRONWALL.

(Read before the American Mathematical Society September 7, 1920.)

In constructing examples of power series bounded in their circle of convergence and having specified convergence defects on the circle, it is frequently useful to consider polynomials of degree $n-1$, such that at each of the $n$th roots of unity, the absolute value of the polynomial is less than or equal to a given constant $M$. Under these conditions, the maximum absolute value of the polynomial inside or on the unit circle is less than $4 M \log n$. $^{*}$

It is the purpose of this note to determine those polynomials where this maximum is as large as possible. The result may be stated in the following theorem.

Theorem. When the polynomial

$$
F(z)=a_{0}+a_{1} z+\cdots+a_{n-1} z^{n-1}
$$

* E. Landau, Bemerkungen zu einer Arbeit des Herrn Carleman, MatheMATISCHE ZeITSCHRIFT, vol. 5 (1919), pp. 147-153. 